University of Nebraska - Lincoln

DigitalCommons@University of Nebraska - Lincoln

Faculty Publications from the Department of Electrical \& Computer Engineering, Department Electrical and Computer Engineering

2010

\title{
A Noncooperative Power Control Game in Multiple-Access Fading Channels with QoS Constraints
}

Deli Qiao

University of Nebraska-Lincoln, dlqiao@ce.ecnu.edu.cn

M. Cenk Gursoy

University of Nebraska-Lincoln, gursoy@engr.unl.edu

Senem Velipasalar

University of Nebraska-Lincoln, velipasa@engr.unl.edu

Follow this and additional works at: https://digitalcommons.unl.edu/electricalengineeringfacpub

Part of the Electrical and Computer Engineering Commons

Qiao, Deli; Cenk Gursoy, M.; and Velipasalar, Senem, "A Noncooperative Power Control Game in MultipleAccess Fading Channels with QoS Constraints" (2010). Faculty Publications from the Department of Electrical and Computer Engineering. 129.

https://digitalcommons.unl.edu/electricalengineeringfacpub/129

This Article is brought to you for free and open access by the Electrical \& Computer Engineering, Department of at DigitalCommons@University of Nebraska - Lincoln. It has been accepted for inclusion in Faculty Publications from the Department of Electrical and Computer Engineering by an authorized administrator of DigitalCommons@University of Nebraska - Lincoln. 


\title{
A Noncooperative Power Control Game in Multiple-Access Fading Channels with QoS Constraints
}

\author{
Deli Qiao, Mustafa Cenk Gursoy, Senem Velipasalar \\ Department of Electrical Engineering \\ University of Nebraska-Lincoln, Lincoln, NE 68588 \\ Email: dqiao726@huskers.unl.edu,gursoy@engr.unl.edu,velipasa@engr.unl.edu
}

\begin{abstract}
In this paper, a game-theoretic analysis for the resource allocation policies in fading multiple-access channels (MAC) in the presence of quality of service $(\mathrm{QoS})$ constraints is performed. Effective capacity, which provides the maximum constant arrival rate, or throughput, that a given service process can support while satisfying statistical delay constraints, is considered in a multiuser scenario. We assume that the channel side information (CSI) is available at both the receiver and transmitters, and the transmitters are selfish, rational with certain QoS constraints and average power limitations. Without the aid of the receiver, we prove that there is always a unique admissible Nash equilibrium of the noncooperative power control game. The Nash equilibrium of the power control game is proved to be always inside the rate region where successive decoding techniques are used at the receiver.
\end{abstract}

\section{INTRODUCTION}

Multiuser channels have been extensively studied over the years from an information-theoretic point of view [1]-[5]. For example, Tse and Hanly [4] characterized the capacity region and optimal resource allocation policies. This approach is powerful and obtains all boundary points. Recently, game theory has been incorporated in the analysis of power control policies [6]-[7]. For instance, Lai and Gamal [7] provided a game-theoric solution to the resource allocation problem in multiaccess fading channels. In this paper, the maximum sum-rate of the capacity region has been shown to be the unique Nash equilibrium, where at most one user with the strongest channel decides to transmit, which is the same to $\mathrm{K}-\mathrm{H}$ algorithm [3]. However, these information theoretical studies have not incorporated the delay and QoS constraints.

In this paper, we consider statistical QoS constraints and propose a noncooperative power control game where the players are the multiple access users. To carry on this analysis, we employ the concept of effective capacity [8], which can be seen as the maximum constant arrival rate that a given time-varying service process can support while satisfying statistical QoS guarantees. Effective capacity formulation uses the large deviations theory and incorporates the statistical QoS constraints by capturing the rate of decay of the buffer occupancy probability for large queue lengths. The analysis and application of effective capacity in various settings has

\footnotetext{
${ }^{1}$ This work was supported by the National Science Foundation under Grants CNS-0834753, and CCF-0917265.
}

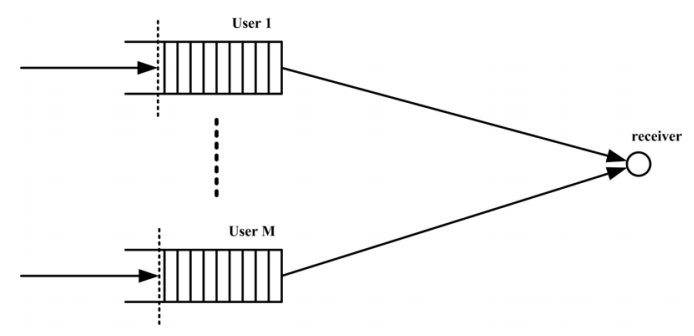

Fig. 1. The system model.

attracted much interest recently (see e.g., [9]-[11] and references therein).

In our proposed game, we assume that the channel state is available at both the transmitters and the receiver. The players are modeled as selfish, rational and are interested in maximizing their own normalized effective capacity, or achievable throughput (rate), subject to an average power constraint. The receiver is not permitted to influence the users in the decisionmaking process. Then, without the aid of the receiver, the users treat the signals of the other users as noise. This worst assumption does not take into consideration that the signal at the receiver can be successively decoded in any order. The unique admissible Nash equilibrium of the game can achieve the maximum sum-rate satisfying each user's QoS constraints for this model, which is no longer the maximal sum-rate point on the achievable region where successive decoding techniques are allowed at the receiver side. Remarkably, while at most one user transmits in previous results [7], all users can transmit simultaneously due to the introduction of QoS constraints.

The paper is organized as follows. Section II provides the preliminaries on multiple-access fading channels, and briefly discusses the concept of effective capacity as a performance metric under statistical QoS constraints. In Section III, we formulate the static noncooperative power control game and propose an optimal resource allocation policy. Finally, Section IV concludes this paper. 


\section{PRELIMINARIES}

\section{A. System Model}

We consider an uplink scenario where $M$ users with individual power constraints and QoS limitations communicate with a single receiver as shown in Fig. 1. It is assumed that the transmitters generate data sequences which are divided into frames of duration $T$. These data frames are initially stored in the buffers before they are transmitted over the wireless channel. The discrete-time signal received at the receiver in the $i^{\text {th }}$ symbol duration is given by

$$
y[i]=\sum_{j=1}^{M} h_{j}[i] x_{j}[i]+n[i], \quad i=1,2, \ldots
$$

where $M$ is the number of users, $x_{j}[i]$ and $h_{j}[i]$ denote the complex-valued channel input and the fading coefficient of the $j$ th user, respectively. We assume that $h_{j}[i] \mathrm{s}$ are jointly stationary and ergodic discrete-time processes, and we denote the magnitude-square of the fading coefficients by $z_{j}[i]=\left|h_{j}[i]\right|^{2}$. The channel input of user $j$ is subject to an average energy constraint $\mathbb{E}\left\{\left|x_{j}[i]\right|^{2}\right\} \leq \bar{P}_{j} / B$ for all $i$, and we assume that the bandwidth available in the system is $B$, so symbol rate is assumed to be $B$ complex symbols per second, indicating that the average power of the system is constrained by $\bar{P} . y[i]$ is the channel output. Above, $n[i]$ is a zero-mean, circularly symmetric, complex Gaussian random variable with variance $\mathbb{E}\left\{|n[i]|^{2}\right\}=N_{0}$. The additive Gaussian noise samples $\{n[i]\}$ are assumed to form an independent and identically distributed (i.i.d.) sequence.

In this work, we suppose that the channel state $\mathbf{z}=$ $\left\{z_{1}, \ldots, z_{M}\right\}$ is available at the both the transmitters and the receiver. We denote the average transmitted signal to noise ratio of user $j$ as $\mathrm{SNR}_{j}=\frac{\bar{P}_{j}}{N_{0} B}$. Also, we denote $P_{j}[i]$ as the instantaneous transmit power in the $i$ th frame. The instantaneous transmitted SNR level for receiver $j$ now becomes $\mu_{j}[i]=\frac{P_{j}[i]}{N_{0} B}$. For a given power allocation policy $\mathcal{U}=\left\{\mu_{1}(\mathbf{z}), \cdots, \mu_{M}(\mathbf{z})\right\}$ where $\mu_{j} \geq 0 \forall j$ can be viewed as a function of $\mathbf{z}$, the achievable rates are described by [4]

$$
\begin{gathered}
\mathcal{R}(\mathcal{U})=\left\{\mathbf{R}: \mathbf{R}(S) \leq \mathbb{E}_{\mathbf{z}}\left\{B \log _{2}\left(1+\sum_{j \in S} \mu_{j}(\mathbf{z}) z_{j}\right)\right\},\right. \\
\forall S \subset\{1, \cdots, M\}\} .
\end{gathered}
$$

Then, the rate region when all transmitters as well as the receiver have CSI is given by

$$
\mathcal{R}_{\mathrm{MAC}}=\bigcup_{\mathcal{U} \in \mathcal{F}} \mathcal{R}(\mathcal{U})
$$

where $\mathcal{F}$ is the set of all feasible power control policies satisfying the average power constraint

$$
\mathcal{F} \equiv\left\{\mathcal{U}: \mathbb{E}_{\mathbf{z}}\left\{\mu_{j}(\mathbf{z}) \leq \operatorname{SNR}_{j}, \mu_{j} \geq 0, \forall j\right\}\right\}
$$

where $\mathrm{SNR}_{j}=\bar{P}_{j} /\left(N_{0} B\right)$ denotes the average transmitted signal-to-noise ratio of user $j$. The maximum instantaneous rate at a given state with any decoding order $\pi$ can be obtained as

$$
R_{\pi(k)}=B \log _{2}\left(1+\frac{\mu_{\pi(k)} z_{\pi(k)}}{1+\sum_{i=k+1}^{M} \mu_{\pi(i)} z_{\pi(i)}}\right) \text { bits/s, } \quad k=1, \cdots, M .
$$

\section{B. Effective Capacity}

In [8], Wu and Negi defined the effective capacity as the maximum constant arrival rate $^{2}$ that a given service process can support in order to guarantee a statistical QoS requirement specified by the QoS exponent $\theta$. If we define $Q$ as the stationary queue length, then $\theta$ is the decay rate of the tail distribution of the queue length $Q$ :

$$
\lim _{q \rightarrow \infty} \frac{\log P(Q \geq q)}{q}=-\theta .
$$

Therefore, for large $q_{\max }$, we have the following approximation for the buffer violation probability: $P\left(Q \geq q_{\max }\right) \approx$ $e^{-\theta q_{\max }}$. Hence, while larger $\theta$ corresponds to more strict QoS constraints, smaller $\theta$ implies looser QoS guarantees. Similarly, if $D$ denotes the steady-state delay experienced in the buffer, then $P\left(D \geq d_{\max }\right) \approx e^{-\theta \delta d_{\max }}$ for large $d_{\max }$, where $\delta$ is determined by the arrival and service processes [10].

The effective capacity is given by

$$
C(\theta)=-\frac{\Lambda(-\theta)}{\theta}=-\lim _{t \rightarrow \infty} \frac{1}{\theta t} \log _{e} \mathbb{E}\left\{e^{-\theta S[t]}\right\} \quad \text { bits/s }
$$

where $S[t]=\sum_{i=1}^{t} s[i]$ is the time-accumulated service process, and $\{s[i], i=1,2, \ldots\}$ denote the discrete-time stationary and ergodic stochastic service process.

In this paper, in order to simplify the analysis while considering general fading distributions, we assume that the fading coefficients stay constant over the frame duration $T$ and vary independently for each frame and each user. In this scenario, $s[i]=T R[i]$, where $R[i]$ is the instantaneous service rate in the $i$ th frame duration $[i T ;(i+1) T]$. Then, (7) can be written as

$$
C(\theta)=-\frac{1}{\theta T} \log _{e} \mathbb{E}_{\mathbf{z}}\left\{e^{-\theta T R[i]}\right\} \quad \text { bits/s }
$$

where $R[i]$ denotes the instantaneous rate sequence varying with $\mathbf{z}$. The effective capacity normalized by bandwidth $B$ is

$$
\mathrm{C}(\theta)=\frac{C(\theta)}{B} \quad \text { bits } / \mathrm{s} / \mathrm{Hz} \text {. }
$$

\section{The Power Control GAme}

In this Section, we first consider the two-user case. Suppose that $\left(\theta_{1}, \theta_{2}\right)$ is a vector composed of the QoS constraint for the two users. Then, $\beta_{j}=\frac{\theta_{j} T B}{\log _{e} 2}, j=1,2$ denotes the corresponding normalized QoS constraint. We assume that the QoS constraints and the power constraints of the users are known by each user.

In the static noncooperative game, the strategy of user $j$ is the power control policy $\mu_{j}(\mathbf{z})$. We choose the normalized

${ }^{2}$ For time-varying arrival rates, effective capacity specifies the effective bandwidth of the arrival process that can be supported by the channel. 
effective capacity $\mathrm{C}_{j}(\mathcal{U})$ as the corresponding payoff. The goal of user $j$ is

$$
\max _{\mu_{j}} \mathrm{C}_{j}\left(\mu_{j}, \mu_{-j}\right) \quad \text { s.t. } \quad \mu_{j} \in \mathcal{F}_{j}
$$

where $\mathcal{F}$ has been given in (4), and $\mu_{-j}$ denotes the power control policies of the other users. Since the base station is not a player of the game, we assume that each user will treat the signal of the other user as interference, and users have no information about the power control policy of the others. For a given power control policy $\mu_{2}\left(z_{1}, z_{2}\right)$ of user 2 , the payoff of user 1 is given by

$$
\begin{gathered}
C_{1}(\mathcal{U})=-\frac{1}{\theta_{1} T B} \log _{e} \mathbb{E}\left\{e^{-\theta_{1} T B \log _{2}\left(1+\frac{\mu_{1}\left(z_{1}, z_{2}\right)}{1+\mu_{2}\left(z_{1}, z_{2}\right) z_{2}}\right)}\right\} \\
=-\frac{1}{\theta_{1} T B} \log _{e}\left(\int_{0}^{\infty} \int_{0}^{\infty}\left(1+\frac{\mu_{1}\left(z_{1}, z_{2}\right) z_{1}}{1+\mu_{2}\left(z_{1}, z_{2}\right) z_{2}}\right)^{-\beta_{1}}\right. \\
\left.p_{\mathbf{z}}\left(z_{1}, z_{2}\right) d z_{1} d z_{2}\right)
\end{gathered}
$$

where $p_{\mathbf{z}}\left(z_{1}, z_{2}\right)$ is the joint probability density function of the channel state. The payoff for user 2 is chosen similarly. Obviously, the payoff of each user depends on the two strategies $\left(\mu_{1}\left(z_{1}, z_{2}\right), \mu_{2}\left(z_{1}, z_{2}\right)\right)$. First, the following definition is needed for the ensuing analysis [12].

Definition 1: A Nash equilibrium is a policy pair $\left(\mu_{1}^{*}, \mu_{2}^{*}\right)$ such that

$$
\begin{aligned}
& \mathrm{C}_{1}\left(\mu_{1}^{*}, \mu_{2}^{*}\right) \geq \mathrm{C}_{1}\left(\mu_{1}^{\prime}, \mu_{2}^{*}\right), \forall \mu_{1}^{\prime} \in \mathcal{F}_{1} \\
& \mathrm{C}_{2}\left(\mu_{1}^{*}, \mu_{2}^{*}\right) \geq \mathrm{C}_{2}\left(\mu_{1}^{*}, \mu_{2}^{\prime}\right), \forall \mu_{2}^{\prime} \in \mathcal{F}_{2} .
\end{aligned}
$$

As indicated by the definition, no user can benefit by deviating from the optimal strategy individually. For any fixed power policy $\mu_{2}\left(z_{1}, z_{2}\right)$, the optimal strategy of user 1 is the solution to the following maximization problem

$$
\begin{gathered}
\mathrm{C}_{1}=\max _{\mu_{1}}-\frac{1}{\theta_{1} T B} \log _{e}\left(\int_{0}^{\infty} \int_{0}^{\infty}\left(1+\frac{\mu_{1}\left(z_{1}, z_{2}\right) z_{1}}{1+\mu_{2}\left(z_{1}, z_{2}\right) z_{2}}\right)^{-\beta_{1}}\right. \\
\left.\cdot p_{\mathbf{z}}\left(z_{1}, z_{2}\right) d z_{1} d z_{2}\right), \\
\text { s.t. } \int_{0}^{\infty} \int_{0}^{\infty} \mu_{1}\left(z_{1}, z_{2}\right) p_{\mathbf{z}}\left(z_{1}, z_{2}\right) d z_{1} d z_{2} \leq \operatorname{SNR}_{1}, \mu_{1}\left(z_{1}, z_{2}\right) \geq 0
\end{gathered}
$$

which can be further reduced to the following minimization problem

$$
\begin{aligned}
\min _{\mu_{1}} & \int_{0}^{\infty} \int_{0}^{\infty}\left(1+\frac{\mu_{1}\left(z_{1}, z_{2}\right) z_{1}}{1+\mu_{2}\left(z_{1}, z_{2}\right) z_{2}}\right)^{-\beta_{1}} p_{\mathbf{z}}\left(z_{1}, z_{2}\right) d z_{1} d z_{2} \\
\text { s.t. } & \int_{0}^{\infty} \int_{0}^{\infty} \mu_{1}\left(z_{1}, z_{2}\right) p_{\mathbf{z}}\left(z_{1}, z_{2}\right) d z_{1} d z_{2} \leq \mathrm{SNR}_{1} \\
& \mu_{1}\left(z_{1}, z_{2}\right) \geq 0
\end{aligned}
$$

The solution to the above optimization problem is the power allocation (similarly as in [9])

$\mu_{1}\left(z_{1}, z_{2}\right)=\left(\frac{\left(1+\mu_{2}\left(z_{1}, z_{2}\right) z_{2}\right)^{\frac{\beta_{1}}{\beta_{1}+1}}}{\alpha_{1}^{\frac{1}{\beta_{1}+1}} z_{1}^{\frac{\beta_{1}}{\beta_{1}+1}}}-\frac{1+\mu_{2}\left(z_{1}, z_{2}\right) z_{2}}{z_{1}}\right)$

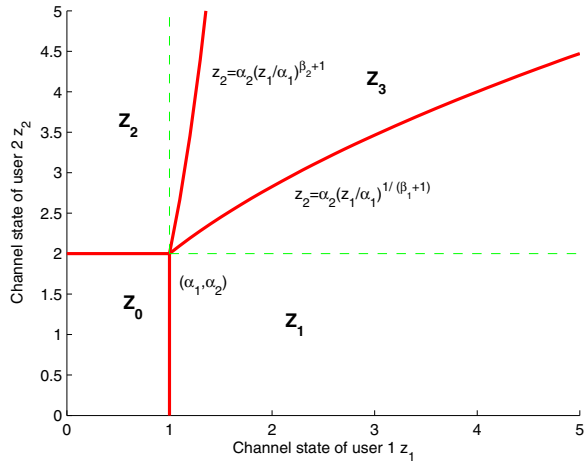

Fig. 2. The strategy regions of different number of users transmitting for the two-user case.

$$
=\frac{1+\mu_{2}\left(z_{1}, z_{2}\right) z_{2}}{z_{1}}\left(\left(\frac{z_{1}}{\alpha_{1}\left(1+\mu_{2}\left(z_{1}, z_{2}\right) z_{2}\right)}\right)^{\frac{1}{\beta_{1}+1}}-1\right)^{+}
$$

where $(x)^{+}=\max \{x, 0\}, \alpha_{1}$ is the threshold chosen to satisfy the following power constraint

$$
\begin{array}{r}
\int_{\alpha_{1}}^{\infty} \int_{0}^{\infty}\left(\frac{\left(1+\mu_{2}\left(z_{1}, z_{2}\right) z_{2}\right)^{\frac{\beta_{1}}{\beta_{1}+1}}}{\alpha_{1}^{\frac{1}{\beta_{1}+1}} z_{1}^{\frac{\beta_{1}}{\beta_{1}+1}}}-\frac{1+\mu_{2}\left(z_{1}, z_{2}\right) z_{2}}{z_{1}}\right)^{+} \\
\cdot p_{\mathbf{z}}\left(z_{1}, z_{2}\right) d z_{2} d z_{1}=\mathrm{SNR}_{1} .
\end{array}
$$

Similarly, the optimal power policy of user 2 can be derived as

$$
\mu_{2}\left(z_{1}, z_{2}\right)=\left(\frac{\left(1+\mu_{1}\left(z_{1}, z_{2}\right) z_{1}\right)^{\frac{\beta_{2}}{\beta_{2}+1}}}{\alpha_{2}^{\frac{1}{\beta_{2}+1}} z_{2}^{\frac{\beta_{2}}{\beta_{2}+1}}}-\frac{1+\mu_{1}\left(z_{1}, z_{2}\right) z_{1}}{z_{2}}\right)^{+}
$$

where $\alpha_{2}$ is the threshold chosen to satisfy the power constraint

$$
\begin{array}{r}
\int_{\alpha_{2}}^{\infty} \int_{0}^{\infty}\left(\frac{\left(1+\mu_{1}\left(z_{1}, z_{2}\right) z_{1}\right)^{\frac{\beta_{2}}{\beta_{2}+1}}}{\alpha_{2}^{\frac{1}{\beta_{2}+1}} z_{2}^{\frac{\beta_{2}}{\beta_{2}+1}}}-\frac{1+\mu_{1}\left(z_{1}, z_{2}\right) z_{1}}{z_{2}}\right)^{+} \\
\cdot p_{\mathbf{z}}\left(z_{1}, z_{2}\right) d z_{1} d z_{2}=\mathrm{SNR}_{2} .
\end{array}
$$

Obviously, the optimal policy of one user depends largely on its assumption of the other user's strategy. According to the assumption, it will adjust its threshold and power control policy to achieve the maximum normalized effective capacity. At the Nash equilibrium, the power constraints of the two users should be satisfied with equality. Considering the expressions (15) and (18), we have the following result.

Proposition 1: For each pair of $\left(\alpha_{1}, \alpha_{2}\right)$, there is a unique pair of strategies $\left(\mu_{1}, \mu_{2}\right)$. The Nash equilibrium, if it exists, of our proposed power game is not the maximum sum rate point of the rate region where superposition coding with successive decoding is employed.

Proof: Please see Appendix A.

According to the proof, there will be a disjoint division of 
the channel state, each region corresponding to a specific set of users transmitting. Fig. 2 shows an example of the partition of the channel state space. If $\mathbf{z}$ lies in region $\mathbf{Z}_{0}$, both users see weak channels compared to the background noise and decide not to transmit. If $\mathbf{z}$ lies in region $\mathbf{Z}_{1}$ (or $\mathbf{Z}_{2}$ ), user 2 (or user 1 , respectively) decides that the guessed interference of the other user plus the noise is so high that it decides not to transmit and conserve the power. When $\mathbf{z}$ falls into region $\mathbf{Z}_{3}$, both users see a relatively weak interference from the other user and allocate power according the best strategy. The power level in this area is a solution to (29) and (30), based on the assumption that the two users are rational.

Obviously, for each pair of $\left(\alpha_{1}, \alpha_{2}\right)$, the power strategy for each user is unique. Then, we have the following results.

Proposition 2: There is always a Nash equilibrium that can maximize the sum-rate throughput. And the optimal power control policies of the two users are given in (20) and (21) on the next page where $\left(\mu_{1}^{*}\left(z_{1}, z_{2}\right), \mu_{2}^{*}\left(z_{1}, z_{2}\right)\right)$ is the pair of power allocation policies solving (29) with a given $\left(\alpha_{1}^{*}, \alpha_{2} *\right)$ satisfying the power constraints, which can be derived numerically.

Proof: Please see Appendix B.

We have proved the existence of the Nash equilibrium. Before proceeding any further, we take advantage of the concept of admissible Nash equilibrium ([12, Definition 3.3]).

Definition 2: A Nash equilibrium strategy pair $\left(\mu_{1}^{*}, \mu_{2}^{*}\right)$ is said to be admissible if there exists no other Nash equilibrium strategy pair $\left(\mu_{1}^{\prime}, \mu_{2}^{\prime}\right)$ such that $C_{1}\left(\mu_{1}^{\prime}, \mu_{2}^{\prime}\right) \geq$ $\mathrm{C}_{1}\left(\mu_{1}^{*}, \mu_{2}^{*}\right), \mathrm{C}_{2}\left(\mu_{1}^{\prime}, \mu_{2}^{\prime}\right) \geq \mathrm{C}_{2}\left(\mu_{1}^{*}, \mu_{2}^{*}\right)$, and at least one of the equalities is strict.

With this notation, we have the following result.

Proposition 3: There is always a unique admissible Nash equilibrium which maximizes the sum-rate.

Proof: Please see Appendix C.

Then, the algorithm stated in Proposition 2 will obviously reach the unique admissible Nash equilibrium if the two users agree on starting the iteration with the same large enough threshold. The two users will only need to compute the threshold pair $\left(\alpha_{1}^{*}, \alpha_{2}^{*}\right)$ off-line according to the channel statistics and the complete information of the other user. In the example, we choose $\mathrm{SNR}_{1}=-12.33 \mathrm{~dB}, \mathrm{SNR}_{2}=-23.67 \mathrm{~dB}$, $\beta_{1}=1, \beta_{2}=2$. At the Nash Equilibrium, $\alpha_{1}^{*}=1.0025, \alpha_{2}^{*}=$ $1.9994, \mathrm{C}_{1}=0.1329 \mathrm{bps} / \mathrm{Hz}, \mathrm{C}_{2}=0.0165 \mathrm{bps} / \mathrm{Hz}$. The power allocation of the two users is shown in Fig. 3. With the same condition, when power control is allowed, $(0.1318,0.0172)$ is achievable if the users are decoded in the order $(1,2)$, and $(0.1330,0.0169)$ is achievable if the users are decoded in the order $(2,1)$ according to the optimal policies given in [14]. Obviously, the Nash equilibrium in the presence of QoS constraints is no longer the maximal sum-rate point of the achievable rate region where successive decoding is used. If each user perform the power control policy for singleinput single-output specified in [9], the point achieved is $(0.1317,0.0159)$, the sum of which is less than that obtained at Nash equilibrium.

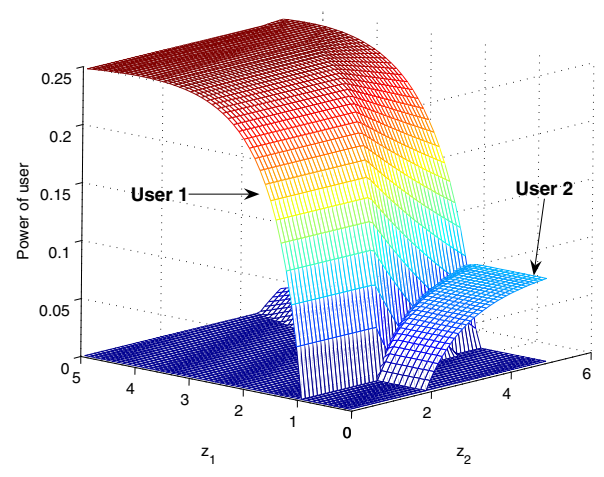

Fig. 3. The power allocation for the two-user case.

\section{CONCLUSION}

In this paper, we have characterized the noncooperative power control policy of two-user multiple-access fading channels by considering effective capacity. We have shown that at most two users will be transmitting due to the introduction of QoS constraints, and hence the maximum sum-rate is always less than the one achieved if successive decoding techniques are considered. We have provided the optimal power control policies at the Nash equilibrium. In particular, we have proved the uniqueness of the admissible Nash equilibrium of our proposed game. Numerical results in consistence with our analysis are provided as well.

\section{APPENDIX}

\section{A. Proof for Proposition 1}

With (16), we can find that if user 1 decides to transmit, or $\mu_{1}\left(z_{1}, z_{2}\right)>0$, we should have

$$
\frac{z_{1}}{\alpha_{1}\left(1+\mu_{2}\left(z_{1}, z_{2}\right) z_{2}\right)}>1 \text {. }
$$

This implies that when $\mu_{1}\left(z_{1}, z_{2}\right)>0$, the channel state of user 1 normalized by the background noise and the interference from user 2 should be greater than the threshold. Similarly, we can derive the condition for user 2 to allocate power to the current state as

$$
\frac{z_{2}}{\alpha_{2}\left(1+\mu_{1}\left(z_{1}, z_{2}\right) z_{1}\right)}>1 \text {. }
$$

Therefore, it is possible that both of the conditions (22) and (23) can be satisfied simultaneously. Then, we will have at most two users transmitting at the same time. Although implicitly, we assume that the pair $\left(\alpha_{1}, \alpha_{2}\right)$ exist.

We can see that when only user 1 transmits, we have $\mu_{2}\left(z_{1}, z_{2}\right)=0$, and condition (22) can be satisfied while (23) cannot. According to (15), we can find that

$$
\mu_{1}\left(z_{1}, z_{2}\right)=\frac{1}{\alpha_{1}^{\frac{1}{\beta_{1}+1}} z_{1}^{\frac{\beta_{1}+1}{\beta_{1}+1}}}-\frac{1}{z_{1}} .
$$

Substituting $\mu_{1}\left(z_{1}, z_{2}\right)$ and $\mu_{2}\left(z_{1}, z_{2}\right)$ into (22) and (23), we 


$$
\begin{aligned}
& \mu_{1}^{*}= \begin{cases}\frac{1}{\alpha_{1}^{* \frac{1}{\beta_{1}+1}} z_{1}^{\beta_{1}+1}}-\frac{1}{z_{1}}, & z_{1}>\alpha_{1}^{*} \& \frac{z_{2}}{\alpha_{2}^{*}} \leq\left(\frac{z_{1}}{\alpha_{1}^{*}}\right)^{\frac{1}{\beta_{1}+1}} \\
\mu_{1}^{*}\left(z_{1}, z_{2}\right), & z_{1}>\alpha_{1}^{*} \&\left(\frac{z_{1}}{\alpha_{1}^{*}}\right)^{\frac{1}{\beta_{1}+1}}<\frac{z_{2}}{\alpha_{2}^{*}}<\left(\frac{z_{1}}{\alpha_{1}^{*}}\right)^{\beta_{2}+1} \\
0, & \text { otherwise }\end{cases} \\
& \mu_{2}^{*}= \begin{cases}\frac{1}{\alpha_{2}^{*} \frac{1}{\beta_{2}+1} z_{2}^{\frac{\beta_{2}}{\beta_{2}+1}}}-\frac{1}{z_{2}}, & z_{2}>\alpha_{2}^{*} \& \frac{z_{1}}{\alpha_{1}^{*}} \leq\left(\frac{z_{2}}{\alpha_{2}^{*}}\right)^{\frac{1}{\beta_{2}+1}} \\
\mu_{2}^{*}\left(z_{1}, z_{2}\right), & z_{2}>\alpha_{2}^{*} \&\left(\frac{z_{2}}{\alpha_{2}^{*}}\right)^{\frac{1}{\beta_{2}+1}}<\frac{z_{1}}{\alpha_{1}^{*}}<\left(\frac{z_{2}}{\alpha_{2}^{*}}\right)^{\beta_{1}+1} \\
0, & \text { otherwise }\end{cases}
\end{aligned}
$$

have

$$
\mathbf{Z}_{1}=\left\{\mathbf{z}: z_{1}>\alpha_{1}, \frac{z_{2}}{\alpha_{2}} \leq\left(\frac{z_{1}}{\alpha_{1}}\right)^{\frac{1}{\beta_{1}+1}}\right\}
$$

which specifies the region in which only user 1 transmits. Similarly, when only user 2 transmits, we have $\mu_{1}\left(z_{1}, z_{2}\right)=0$. $\mu_{2}\left(z_{1}, z_{2}\right)$ is given by

$$
\mu_{2}\left(z_{1}, z_{2}\right)=\frac{1}{\alpha_{2}^{\frac{1}{\beta_{2}+1}} z_{2}^{\frac{\beta_{2}}{\beta_{2}+1}}}-\frac{1}{z_{2}}
$$

in the region confined by

$$
\mathbf{Z}_{2}=\left\{\mathbf{z}: z_{2}>\alpha_{2}, \frac{z_{1}}{\alpha_{1}} \leq\left(\frac{z_{2}}{\alpha_{2}}\right)^{\frac{1}{\beta_{2}+1}}\right\} .
$$

When both users transmit, (22) and (23) can be satisfied. We can easily see that $\mu_{1}\left(z_{1}, z_{2}\right)$ is upperbounded by $\frac{\frac{z_{2}}{\alpha_{2}}-1}{z_{1}}$ and $\mu_{2}\left(z_{1}, z_{2}\right)$ is upperbounded by $\frac{\frac{z_{1}}{\alpha_{1}}-1}{z_{2}}$. From previous discussion, this region lies in

$$
\mathbf{Z}_{3}=\left\{\mathbf{z}: z_{1}>\alpha_{1},\left(\frac{z_{1}}{\alpha_{1}}\right)^{\frac{1}{\beta_{1}+1}}<\frac{z_{2}}{\alpha_{2}}<\left(\frac{z_{1}}{\alpha_{1}}\right)^{\beta_{2}+1}\right\} \text {. }
$$

We will have

$$
\left\{\begin{array}{l}
\mu_{1}\left(z_{1}, z_{2}\right)=\frac{\left(1+\mu_{2}\left(z_{1}, z_{2}\right) z_{2}\right)^{\frac{\beta_{1}}{\beta_{1}+1}}}{\alpha_{1}^{\frac{1}{\beta_{1}+1}} z_{1}^{\frac{\beta_{1}}{\beta_{1}+1}}}-\frac{1+\mu_{2}\left(z_{1}, z_{2}\right) z_{2}}{z_{1}} \\
\mu_{2}\left(z_{1}, z_{2}\right)=\frac{\left(1+\mu_{1}\left(z_{1}, z_{2}\right) z_{1}\right)^{\frac{\beta_{2}}{\beta_{2}+1}}}{\alpha_{2}^{\frac{\beta_{2}+1}{\beta_{2}}} z_{2}^{\frac{\beta_{2}+1}{\beta_{2}}}}-\frac{1+\mu_{1}\left(z_{1}, z_{2}\right) z_{1}}{z_{2}}
\end{array}\right.
$$

which can be written as

$\left\{\begin{array}{l}1+\mu_{1}\left(z_{1}, z_{2}\right) z_{1}+\mu_{2}\left(z_{1}, z_{2}\right) z_{2}=\left(\frac{z_{1}}{\alpha_{1}}\right)^{\frac{1}{\beta_{1}+1}}\left(1+\mu_{2}\left(z_{1}, z_{2}\right) z_{2}\right)^{\frac{\beta_{1}}{\beta_{1}+1}} \\ 1+\mu_{1}\left(z_{1}, z_{2}\right) z_{1}+\mu_{2}\left(z_{1}, z_{2}\right) z_{2}=\left(\frac{z_{2}}{\alpha_{2}}\right)^{\frac{1}{\beta_{2}+1}}\left(1+\mu_{1}\left(z_{1}, z_{2}\right) z_{1}\right)^{\frac{\beta_{2}}{\beta_{2}+1}}\end{array}\right.$

Given $\alpha_{1}, \alpha_{2}, z_{1}, z_{2}$, we can find from (29) that $\mu_{1}\left(z_{1}, z_{2}\right)$ is a concave function of $\mu_{2}\left(z_{1}, z_{2}\right)$ and $\mu_{2}\left(z_{1}, z_{2}\right)$ is a concave function of $\mu_{1}\left(z_{1}, z_{2}\right)$. The intersections of these two curves in the $\mu_{1}-\mu_{2}$ plane denote the solutions satisfying the equations. Consider first the function $\mu_{1}\left(z_{1}, z_{2}\right)$ of $\mu_{2}\left(z_{1}, z_{2}\right)$, the definition domain to ensure $\mu_{1}\left(z_{1}, z_{2}\right)>0$ is $\mu_{2}\left(z_{1}, z_{2}\right) \in$ $\left(0, \frac{\frac{z_{1}}{\alpha_{1}}-1}{z_{2}}\right)$. The curve intersects with $\mu_{2}=0$ at

$$
\mu_{1}\left(z_{1}, z_{2}\right)=\frac{1}{\alpha_{1}^{\frac{1}{\beta_{1}+1}} z_{1}^{\frac{\beta_{1}}{\beta_{1}+1}}}-\frac{1}{z_{1}}>0 .
$$

Similarly, the definition domain for function $\mu_{2}\left(z_{1}, z_{2}\right)$ of $\mu_{1}\left(z_{1}, z_{2}\right)$ is $\mu_{1}\left(z_{1}, z_{2}\right) \in\left(0, \frac{\frac{z_{2}}{\alpha_{2}}-1}{z_{1}}\right)$. The curve intersects with $\mu_{1}=0$ at

$$
\mu_{2}\left(z_{1}, z_{2}\right)=\frac{1}{\alpha_{2}^{\frac{1}{\beta_{2}+1}} z_{2}^{\frac{\beta_{2}}{\beta_{2}+1}}}-\frac{1}{z_{2}}>0 .
$$

Then, there will be at least one intersection between the two curves. Denote $\left(\mu_{1}^{2}\left(z_{1}, z_{2}\right), \mu_{2}^{2}\left(z_{1}, z_{2}\right)\right)$ as one intersection.

Suppose there is another point $\left(\mu_{1}^{\prime 2}\left(z_{1}, z_{2}\right), \mu_{2}^{\prime 2}\left(z_{1}, z_{2}\right)\right)$ satisfying (29). Without loss of generality, we assume $\mu_{1}^{\prime 2}\left(z_{1}, z_{2}\right)<\mu_{1}^{2}\left(z_{1}, z_{2}\right)$. According to the first equation in (30), we can find that $\mu_{2}^{\prime 2}\left(z_{1}, z_{2}\right)<\mu_{2}^{2}\left(z_{1}, z_{2}\right)$ as well. Then, these two points are on a straight line $l$ with positive slope in the $\mu_{1}-\mu_{2}$ plane. Due to the concavity of the curve, if the interception of $l$ on $\mu_{1}=0$ is less than 0 , then the curve for $\mu_{2}\left(z_{1}, z_{2}\right)$ as a function of $\mu_{1}\left(z_{1}, z_{2}\right)$ intersects with $\mu_{1}=0$ at a negative value, i.e., $\mu_{2}\left(z_{1}, z_{2}\right)<0$ at $\mu_{1}\left(z_{1}, z_{2}\right)=0$. This violates (32). Similarly, if the interception of $l$ on $\mu_{2}=0$ is less than 0 , we can derive that $\mu_{1}\left(z_{1}, z_{2}\right)<0$ at $\mu_{2}\left(z_{1}, z_{2}\right)=0$, violating (31). Thus, there is only one solution to the equations, i.e., there is a unique pair of $\left(\mu_{1}, \mu_{2}\right)$ for a given pair $\left(\alpha_{1}, \alpha_{2}\right)$.

From the above discussion, we can see that at most two users will be transmitting at the same time, and hence the sumrate throughput at the Nash equilibrium is always suboptimal to the one derived when the superposition coding technique with successive decoding is used, where there is always at least one user viewing no interference from the other users. $\square$

\section{B. Proof for Proposition 2}

We can rewrite (17) and (19) as

$$
\begin{aligned}
\iint_{\mathbf{z}_{1}} & \left(\frac{1}{\alpha_{1}^{\frac{1}{\beta_{1}+1}} z_{1}^{\frac{\beta_{1}}{\beta_{1}+1}}}-\frac{1}{z_{1}}\right) p_{\mathbf{z}}\left(z_{1}, z_{2}\right) d z_{2} d z_{1} \\
& +\iint_{\mathbf{z}_{3}} \mu_{1}^{2}\left(z_{1}, z_{2}\right) p_{\mathbf{z}}\left(z_{1}, z_{2}\right) d z_{2} d z_{1}=\mathrm{SNR}_{1},
\end{aligned}
$$




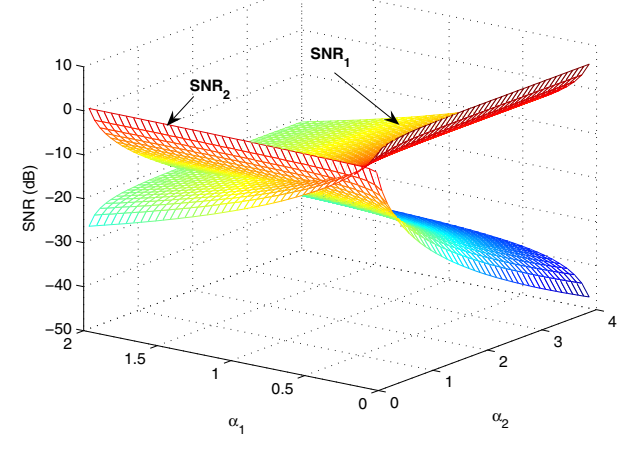

Fig. 4. The average SNRs with respect to $\alpha_{1}$ and $\alpha_{2}$ in Rayleigh fading channel.

$$
\begin{aligned}
\iint_{\mathbf{z}_{2}} & \left(\frac{1}{\alpha_{2}^{\frac{1}{\beta_{2}+1}} z_{1}^{\frac{\beta_{2}}{\beta_{2}+1}}}-\frac{1}{z_{2}}\right) p_{\mathbf{z}}\left(z_{1}, z_{2}\right) d z_{2} d z_{1} \\
& +\iint_{\mathbf{z}_{3}} \mu_{2}^{2}\left(z_{1}, z_{2}\right) p_{\mathbf{z}}\left(z_{1}, z_{2}\right) d z_{2} d z_{1}=\mathrm{SNR}_{2} .
\end{aligned}
$$

Combining previous discussions on the division of the rate regions and the power allocation strategy, we plot $\mathrm{SNR}_{1}$ and $\mathrm{SNR}_{2}$ as functions of $\alpha_{1}$ and $\alpha_{2}$ in the Rayleigh fading channel with unit expected value in Fig. 4 as an example. It can be easily found that $\mathrm{SNR}_{1}$ is a nonincreasing function of $\alpha_{1}$, and a nondecreasing function of $\alpha_{2}$, while $\mu_{2}\left(z_{1}, z_{2}\right)$ is a nonincreasing function of $\alpha_{2}$, and a nondecreasing function of $\alpha_{1}$. Then, for any two threshold pairs $\left(\alpha_{1}^{\prime}, \alpha_{2}^{\prime}\right)$ and $\left(\alpha_{1}^{*}, \alpha_{2}^{*}\right)$ with $\alpha_{1}^{\prime}=$ $\alpha_{1}^{*}, \alpha_{2}^{\prime} \geq \alpha_{2}^{*}$, we will have $\operatorname{SNR}_{1}\left(\alpha_{1}^{\prime}, \alpha_{2}^{\prime}\right) \geq \operatorname{SNR}_{1}\left(\alpha_{1}^{*}, \alpha_{2}^{*}\right)$ and $\operatorname{SNR}_{2}\left(\alpha_{1}^{\prime}, \alpha_{2}^{\prime}\right) \leq \operatorname{SNR}_{2}\left(\alpha_{1}^{*}, \alpha_{2}^{*}\right)$, where $\operatorname{SNR}_{i}\left(\alpha_{1}, \alpha_{2}\right)$ represents the SNR of user $i$ associated with the given threshold pair. Thus, similar to [7], we let $\alpha_{1}(0)=\varepsilon, \alpha_{2}(0)=\varepsilon$ with $\varepsilon$ close to the maximum channel gain that can be achieved, in which case $\operatorname{SNR}_{i}\left(\alpha_{1}(0), \alpha_{2}(0)\right), i=1,2$ are close to 0 . First, fix $\alpha_{1}(n-1)$ and find $\alpha_{2}(n)$ satisfying the average power constraint of user 1 , which is a solution to (34). At this step, we have $\alpha_{2}(n) \leq$ $\alpha_{2}(n-1)$ and $\operatorname{SNR}_{1}\left(\alpha_{1}(n-1), \alpha_{2}(n)\right) \leq \operatorname{SNR}_{1}$. Then, fix $\alpha_{2}(n)$ to find the $\alpha_{1}(n)$ satisfying the average power constraint of user 2, which is a solution to (33). At this step, we have $\alpha_{1}(n) \leq \alpha_{1}(n-1)$ and $\operatorname{SNR}_{2}\left(\alpha_{1}(n), \alpha_{2}(n)\right) \leq \mathrm{SNR}_{2}$. Iteratively, we will obtain nonincreasing sequences $\alpha_{1}(n), \alpha_{2}(n)$ with $\operatorname{SNR}_{1}\left(\alpha_{1}(n), \alpha_{2}(n)\right) \rightarrow \operatorname{SNR}_{1}$ and $\operatorname{SNR}_{2}\left(\alpha_{1}(n), \alpha_{2}(n)\right) \rightarrow$ $\mathrm{SNR}_{2}$. Since $\mathrm{SNR}_{i}, i=1,2$ are limited value, we have that both of the sequences are lowerbounded. Then, there must exist the constants [13]

$$
\begin{aligned}
& \inf _{n} \alpha_{1}(n)=\lim _{n \rightarrow \infty} \alpha_{1}(n)=\alpha_{1}^{*}, \operatorname{SNR}_{1}\left(\alpha_{1}^{*}, \alpha_{2}^{*}\right)=\mathrm{SNR}_{1}, \\
& \inf _{n} \alpha_{2}(n)=\lim _{n \rightarrow \infty} \alpha_{2}(n)=\alpha_{2}^{*}, \operatorname{SNR}_{2}\left(\alpha_{1}^{*}, \alpha_{2}^{*}\right)=\mathrm{SNR}_{2} .
\end{aligned}
$$

Thus, throughout the above process, we have proved that there must be a pair of thresholds $\left(\alpha_{1}^{*}, \alpha_{2}^{*}\right)$ at the Nash equilibrium. And the optimal power policies $\left(\mu_{1}^{*}, \mu_{2}^{*}\right)$ can be derived correspondingly.

\section{Proof for Proposition 3}

Let $\left(\mu_{1}^{*}, \mu_{2}^{*}\right)$ and $\left(\mu_{1}^{\prime}, \mu_{2}^{\prime}\right)$ be the two pairs of power policies at the Nash equilibrium. The corresponding threshold pairs are $\left(\alpha_{1}^{\prime}, \alpha_{2}^{\prime}\right)$ and $\left(\alpha_{1}^{*}, \alpha_{2}^{*}\right)$. These pairs are the solutions to the equations (33) and (34). Combining previous discussion on the increasing and decreasing characteristics of $\mathrm{SNR}_{i}, i=1,2$ in $\alpha_{i}, i=1,2$, we can easily derive that $\alpha_{1}^{*}=\alpha_{1}^{\prime}$ iff $\alpha_{2}^{*}=\alpha_{2}^{\prime}, \alpha_{1}^{*}>\alpha_{1}^{\prime}$ iff $\alpha_{2}^{*}>\alpha_{2}^{\prime}$, and $\alpha_{1}^{*}<\alpha_{1}^{\prime}$ iff $\alpha_{2}^{*}<\alpha_{2}^{\prime}$. Then, the threshold pairs must have a strict order, and we assume that $\alpha_{1}^{*}>\alpha_{1}^{\prime}, \alpha_{2}^{*}>\alpha_{2}^{\prime}$. Then, with the corresponding unique power strategies $\left(\mu_{1}^{*}, \mu_{2}^{*}\right)$ and $\left(\mu_{1}^{\prime}, \mu_{2}^{\prime}\right)$, we can show that $\mathrm{C}_{1}\left(\mu_{1}^{*}, \mu_{2}^{*}\right)>\mathrm{C}_{1}\left(\mu_{1}^{\prime}, \mu_{2}^{\prime}\right)$ and $\mathrm{C}_{2}\left(\mu_{1}^{*}, \mu_{2}^{*}\right)>\mathrm{C}_{2}\left(\mu_{1}^{\prime}, \mu_{2}^{\prime}\right)$. Simply put, with the division of the channel state shown previously, we can see that decreasing the threshold pair will extend the area for $\mathbf{Z}_{3}$, and according to (30), we can find that $\mu_{1}, \mu_{2}$ will increase as well, i.e., both of the users have to allocate more power to compete with the other user. As it turns out, both user see increased interference from the other user, implying that $\mathrm{C}_{1}\left(\mu_{1}^{*}, \mu_{2}^{*}\right)>\mathrm{C}_{1}\left(\mu_{1}^{\prime}, \mu_{2}^{\prime}\right)$ and $\mathrm{C}_{2}\left(\mu_{1}^{*}, \mu_{2}^{*}\right)>\mathrm{C}_{2}\left(\mu_{1}^{\prime}, \mu_{2}^{\prime}\right)$.

Therefore, the Nash equilibrium pairs have strict order and the equilibrium power strategies $\left(\mu_{1}^{*}, \mu_{2}^{*}\right)$ corresponding to the largest threshold pair $\left(\alpha_{1}^{*}, \alpha_{2}^{*}\right)$ will achieve the largest sum rate. Hence, we prove the uniqueness of admissible Nash equilibrium.

\section{REFERENCES}

[1] T. M. Cover and J. A. Thomas, Elements of Information Theory. New York: Wiley, 1991.

[2] L. Ozarow, S. Shamai (Shitz), and A. Wyner, "Information theoretic considerations for cellular mobile radio," IEEE Trans. Veh. Technol., vol. 43, pp. 359-378, May 1994.

[3] R. Knopp and P.A. Humblet, "Information capacity and power control in single-cell multiuser communications," Proc. IEEE Int. Conf. Communication, Seattle, WA, Jun. 1995, vol. 1, pp. 331-335.

[4] D. Tse and S. Hanly, "Multi-access fading channels-Part I: Polymatroid structure, optimal resource allocation and throughput capacities,"IEEE Trans. Inform. Theory, vol. 44, no. 7, pp. 2796-2815, Nov. 1998.

[5] S. Hanly and D. Tse, "Multi-access fading channels-Part II: Delaylimited capacities,"IEEE Trans. Inform. Theory, vol. 44, no. 7, pp. 28162831, Nov. 1998

[6] F. Meshkati, M. Chiang, H.V. Poor, and S.C. Schwartz,"A noncooperative power control game for multicarrier CDMA systems,"IEEE. J. Sel. Areas Commun., vol.24, no.6, pp.1115-1129, Jun. 2006.

[7] L. Lai and H. El Gamal, "The water-filling game in fading multipleaccess channels," IEEE Trans. Inform. Theory, vol. 54, no. 5, pp.21102122, May 2008.

[8] D. Wu and R. Negi "Effective capacity: a wireless link model for support of quality of service," IEEE Trans. Wireless Commun., vol.2,no. 4, pp.630-643. July 2003

[9] J. Tang and X. Zhang, "Quality-of-service driven power and rate adaptation over wireless links," IEEE Trans. Wireless Commun., vol. 6, no. 8, pp. 3058-3068, Aug. 2007.

[10] J. Tang and X. Zhang, "Cross-layer-model based adaptive resource allocation for statistical QoS guarantees in mobile wireless networks," IEEE Trans. Wireless Commun., vol. 7, no. 6, pp.2318-2328, June 2008.

[11] L. Liu, P. Parag, and J.-F. Chamberland, "Quality of service analysis for wireless user-cooperation networks," IEEE Trans. Inform. Theory, vol. 53, no. 10, pp. 3833-3842, Oct. 2007

[12] T. Basar and G. J. Olsder, Dynamic Noncooperative Game Theory, Philadelphia, PA: SIAM, 1998.

[13] W. Rudin, Principles of Mathmatical Analysis, 3rd Ed.. McGraw-Hill Science/Engineering/Math, 1976.

[14] D. Qiao, M.C. Gursoy, and S. Velipasalar, "On the achievable throughput region in the multiple-access fading channels with QoS constraints," to appear in IEEE International Conference on Communications (ICC), May 2010. 